\author{
M.T. Kosmakova ${ }^{1}$, S.A. Iskakov ${ }^{1, *}$, L.Zh. Kasymova ${ }^{1,2}$ \\ ${ }^{1}$ Buketov Karaganda university, Karaganda, Kazakhstan; \\ ${ }^{2}$ Karaganda technical university, Karaganda, Kazakhstan \\ (E-mail: svetlanamir578@gmail.com, isagyndyk@mail.ru,l.kasymova2017@mail.ru)
}

\title{
To solving the fractionally loaded heat equation
}

\begin{abstract}
In this paper we consider a boundary value problem for a fractionally loaded heat equation in the class of continuous functions. Research methods are based on an approach to the study of boundary value problems, based on their reduction to integral equations. The problem is reduced to a Volterra integral equation of the second kind by inverting the differential part. We also carried out a study the limit cases for the fractional derivative order of the term with a load in the heat equation of the boundary value problem. It is shown that the existence and uniqueness of solutions to the integral equation depends on the order of the fractional derivative in the loaded term.
\end{abstract}

Keywords: loaded equation, fractional derivative, heat equation, Volterra integral equation, special function.

\section{Introduction}

The study of fractional differential equations was actively carried out as in previous decades [1-4], and now interest in this area continues to grow [5-7]. This is due both to the development of the fractional integration and differentiation theory, as well as applications of the apparatus of fractional integration and differentiation in various fields of science. The physical interpretation for fractional differential equations was considered in [3] from the point of view of the Riemann-Liouville's derivatives, as well as in [4]. In [6] a boundary value problem with integral conditions is considered for one class of fractional differential equations involving impulses. Some results of the existence of a solution for higher order differential equations with integral conditions can be found in [5]. Also an important section in the theory of differential equations is the class of loaded equations: $K u=L u(x)+M u(x)=f(x)$ in a domain $Q$ from $R^{n}$, where $L$ is a differential operator, and $M$ is a differential or integro-differential operator, including the operation of taking the trace of the function $u(x)$ on manifolds from the closure Q of dimension strictly less than $n$. Solving many important problems, for example, on the optimal management of the agroecosystem, is reduced to the study of such equations. In [8] on numerous examples A.M. Nakhushev showed the practical and theoretical importance of studies on loaded equations. In the papers of M.T. Jenaliev and students of his scientific school, the theory of loaded equations was further developed [9-12]. In [11], [12] loaded differential equations are interpreted as weak or strong perturbations of differential equations.

Of interest are boundary value problems for the fractionally loaded heat equation when the loaded term is presented in the form of a fractional derivative. The goal of papers [13-14] is to clarify the character of the fractional load on the solvability issues of the first boundary value problem for the heat equation, the load moves with a constant velocity. The loaded term is the trace of the fractional order derivative on the manifold $x=t$, namely, the loaded term is represented as a Riemann-Liouville fractional derivative. The resulting Volterra singular integral equation has a nonempty spectrum for certain values of the fractional derivative order. In the papers [15-16] the loaded term is represented in the form of the Caputo fractional derivative with respect to the time variable and the spatial variable, and the order of the derivative in the loaded term is less than the order of the differential part.

In this paper, we study a boundary value problem for a fractionally loaded heat equation (the loaded term of the equation is represented as a Riemann-Liouville fractional derivative, the load moves according to an arbitrary law). The boundary value problem is reduced to a Volterra integral equation of the second kind with a kernel containing a special function, namely, the degenerated hypergeometric Tricomi function. The limiting cases of the order of the fractional derivative in the term with the equation load are also investigated, and continuity in the order of the fractional derivative is shown. The solvability of the integral equation in the class of continuous functions is established depending on the nature of the load for small values of time.

\footnotetext{
${ }^{*}$ Corresponding author.

E-mail: isagyndyk@mail.ru
} 


\section{Basic definitions and some background}

Let us first recall some previously known concepts and results. The first one is the definition of the Riemann-Liouville fractional derivative. follows

Definition 1 ([1]). Let $f(t) \in L_{1}[a, b]$. Then, the Riemann-Liouville derivative of the order $\beta$ is defined as

$$
{ }_{r} D_{a, t}^{\beta} f(t)=\frac{1}{\Gamma(n-\beta)} \frac{d^{n}}{d t^{n}} \int_{a}^{t} \frac{f(\tau)}{(t-\tau)^{\beta-n+1}} d \tau, \quad \beta, a \in R, \quad n-1<\beta<n .
$$

When $a=0, \quad n=1$ we have:

$$
{ }_{r} D_{0, t}^{\beta} f(t)=\frac{1}{\Gamma(1-\beta)} \frac{d}{d t} \int_{0}^{t} \frac{f(t)}{(t-\tau)^{\beta}} d \tau
$$

From formula (1) it follows that

$$
{ }_{r} D_{a, t}^{0} f(t)=f(t), \quad{ }_{r} D_{a, t}^{n} f(t)=f^{(n)}(t), \quad n \in N .
$$

We also give definitions and some properties of special functions that arise in the study of boundary value problem posed in the work.

$\operatorname{erf} z=\frac{2}{\sqrt{\pi}} \int_{0}^{z} \exp \left(-\zeta^{2}\right) d \zeta$ is the integral of probabilities;

$\operatorname{erfc} z=\frac{2}{\sqrt{\pi}} \int_{z}^{\infty} \exp \left(-\zeta^{2}\right) d \zeta$ is the additional integral of probabilities.

Definition $2([17 ; 119])$ Linearly independent solutions of the equation

$$
\left[z D^{2}+(c-z) D-a\right] \omega(z)=0, \quad D=\frac{d}{d z}
$$

are functions $\Phi(a, c ; z)$ and $\Psi(a, c ; z)$, where $\Phi(a, c ; z)$ is the degenerate hypergeometric function:

$$
\Phi(a, c ; z)=1+\frac{a}{c} \frac{z}{1 !}+\frac{a(a+1)}{c(c+1)} \frac{z^{2}}{2 !}+\frac{a(a+1)(a+2)}{c(c+1)(c+2)} \frac{z^{3}}{3 !}+\ldots-
$$

and $\Psi(a, c ; z)$ is Tricomi degenerate hypergeometric function [18; 1072]:

$$
\Psi(a, c ; z)=\frac{\Gamma(1-c)}{\Gamma(a-c+1)} \Phi(a, c ; z)+\frac{\Gamma(c-1)}{\Gamma(a)} z^{1-c} \Phi(a-c+1,2-c ; z) .
$$

Tricomi degenerate hypergeometric function can be represented as an integral ([19; 365], formula 72.2 (7)):

$$
\Psi(a, c ; z)=\frac{1}{\Gamma(a)} \int_{0}^{\infty} \xi^{a-1}(1+\xi)^{c-a-1} e^{-z \xi} d \xi, \quad[\text { Re } a>0] .
$$

For large values $z$, an asymptotic formula holds ([17; 127], formula 4.7 (1)):

$$
\begin{gathered}
\Psi(a, c ; z) \sim z^{-a}{ }_{2} F_{0}\left(a, 1+a-c ;-\frac{1}{z}\right), \\
|z| \rightarrow \infty, \quad|\arg z| \leq \frac{3 \pi}{2}-\epsilon, \quad \epsilon>0,
\end{gathered}
$$

where ${ }_{2} F_{0}\left(a, 1+a-c ;-\frac{1}{z}\right)$ is a generalized hypergeometric series defined by the formula [17; 136]

$$
{ }_{p} F_{q}\left(a_{1}, a_{2}, \ldots, a_{p} ; b_{1}, b_{2}, \ldots, b_{q} ; z\right)=\sum_{k=0}^{\infty} \frac{\left(a_{1}\right)_{k}\left(a_{2}\right)_{k} \ldots\left(a_{p}\right)_{k}}{\left(b_{1}\right)_{k}\left(b_{2}\right)_{k} \ldots\left(b_{q}\right)_{k}} \frac{z^{k}}{k !},
$$

where

$$
(a)_{k}=\frac{\Gamma(a+k)}{\Gamma(a)}
$$

is the Pohammer symbol. 
Degenerate hypergeometric functions for some values of its arguments are related to a parabolic cylinder function (Weber function) $D_{\nu}(z)[17 ; 212]$

$$
\begin{gathered}
D_{\nu}(z)=2^{\frac{\nu-1}{2}} e^{-\frac{z^{2}}{4}} \Psi\left(\frac{1-\nu}{2}, \frac{3}{2} ; \frac{z^{2}}{2}\right)= \\
=2^{\frac{\nu}{2}} e^{-\frac{z^{2}}{4}}\left\{\frac{\sqrt{\pi}}{\Gamma\left(\frac{1-\nu}{2}\right)} \Phi\left(-\frac{\nu}{2} ; \frac{1}{2} ; \frac{z^{2}}{2}\right)-\frac{\sqrt{2 \pi} z}{\Gamma\left(-\frac{\nu}{2}\right)} \Phi\left(\frac{1-\nu}{2} ; \frac{3}{2} ; \frac{z^{2}}{2}\right)\right\} .
\end{gathered}
$$

Formula (6) is the definition of a parabolic cylinder function $D_{\nu}(z)$.

There is also a representation of the Tricomi degenerate hypergeometric function in terms of the Whittaker function $W_{\lambda, \mu}(z)([19 ; 264]$ formula 2.20$)$ :

$$
\Psi(a, b ; z)=z^{-\frac{b}{2}} e^{\frac{z}{2}} W_{\frac{b}{2}-a, \frac{b-1}{2}}(z) .
$$

For the function $W_{\lambda, \mu}(z) \quad z=0$ is a branch point, a $z=\infty$ is an essentially singular point [18; 1074]. Therefore, we will consider this function only for $|\arg z|<\pi$.

The natural development of fractional calculus is the theory of differential equations with fractional derivatives. At the first stage of the study, we will use the method of integral equations, in which the boundary value problem is reduced to solving the corresponding integral equation with further transformation of the kernel of the obtained equation. Such methods make it possible to formulate boundary value problems more compactly than differential equations, taking into account all the conditions of the problem.

The considered problem is reduced to an integral equation by inverting the differential part.

It's known $[20 ; 57]$ that in the domain $Q=\{(x, t) \mid x>0, \quad t>0\}$ the solution to the boundary value problem of heat conduction

$$
\begin{gathered}
u_{t}=a^{2} u_{x x}+F(x, t), \\
\left.u\right|_{t=0}=f(x),\left.\quad u\right|_{x=0}=g(x),
\end{gathered}
$$

is described by the formula

$$
\begin{aligned}
u(x, t)= & \int_{0}^{\infty} G(x, \xi, t) f(\xi) d \xi+\int_{0}^{t} H(x, t-\tau) g(\tau) d \tau+ \\
& +\int_{0}^{t} \int_{0}^{\infty} G(x, \xi, t-\tau) F(\xi, \tau) d \xi d \tau
\end{aligned}
$$

where

$$
\begin{gathered}
G(x, \xi, t)=\frac{1}{2 \sqrt{\pi a t}}\left\{\exp \left(-\frac{(x-\xi)^{2}}{4 a t}\right)-\exp \left(-\frac{(x+\xi)^{2}}{4 a t}\right)\right\}, \\
H(x, t)=\frac{1}{2 \sqrt{\pi a} t^{3 / 2}} \exp \left(-\frac{x^{2}}{4 a t}\right) .
\end{gathered}
$$

The Green function $G(x, \xi, t-\tau)$ satisfies the relation

$$
\int_{0}^{\infty} G(x, \xi, t-\tau) d \xi=\operatorname{erf}\left(\frac{x}{2 \sqrt{t-\tau}}\right) .
$$

2 Statement of the fractionally loaded boundary value problem of heat conduction

In the domain $Q=\{(x, t) \mid x>0, \quad t>0\}$ we consider the problem

$$
\begin{gathered}
u_{t}-u_{x x}+\left.\lambda\left\{{ }_{r} D_{0, t} u(x, t)\right\}\right|_{x=\gamma(t)}=f(x, t), \\
\left.u\right|_{t=0}=0,\left.u\right|_{x=0}=0,
\end{gathered}
$$

where $\lambda$ is a complex parameter, ${ }_{r} D_{0, t}^{\beta} u(x, t)$ is the Riemann- Liouville derivative (2) of an order $\beta, 0<\beta<1$, $\gamma(t)$ is a continuous increasing function, $\gamma(0)=0$.

The problem is studied in the class of continuous functions. 
3 Reducing the boundary value problem to an integral equation

Lemma 1. The boundary value problem (10)-(11) is equivalently reduced to a Volterra integral equation of the second kind with a kernel that contains a special function.

Proof. We invert the differential part of problem (10)-(11) by formula (8):

$$
\begin{gathered}
u(x, t)=-\left.\lambda \int_{0}^{t} \int_{0}^{\infty} G(x, \xi, t-\tau)\left\{\frac{1}{\Gamma(1-\beta)} \frac{d}{d \tau} \int_{0}^{\tau} \frac{u(\xi, \theta)}{(\tau-\theta)^{\beta}} d \theta\right\}\right|_{\xi=\gamma(\tau)} d \xi d \tau+ \\
+\int_{0}^{t} \int_{0}^{\infty} G(x, \xi, t-\tau) f(\xi, \tau) d \xi d \tau .
\end{gathered}
$$

Taking into account relation (9) and introducing the notation

$$
f_{1}(x, t)=\int_{0}^{t} \int_{0}^{\infty} G(x, \xi, t-\tau) f(\xi, \tau) d \tau
$$

we get the following representation of the solution to the problem (10)-(11):

$$
u(x, t)=-\lambda \int_{0}^{t} \operatorname{erf}\left(\frac{x}{2 \sqrt{t-\tau}}\right) \mu(\tau) d \tau+f_{1}(x, t),
$$

where

$$
\mu(t)=\left.{ }_{r} D_{0, t}^{\beta} u(\xi, t)\right|_{\xi=\gamma(t)}=\left.\left\{\frac{1}{\Gamma(1-\beta)} \frac{d}{d \tau} \int_{0}^{\tau} \frac{u(\xi, \theta)}{(\tau-\theta)^{\beta}} d \theta\right\}\right|_{\xi=\gamma(\tau)} .
$$

From (13) we take the derivative of the order $\beta$ with respect to the variables $t$ on both sides and put $x=\gamma(t)$. On the left side, we get the function $\mu(t)$. We also introduce the notation according to formula (14)

$$
f_{2}(t)=\left.{ }_{r} D_{0, t}^{\beta} f_{1}(x, t)\right|_{x=\gamma(t)}=\left.\frac{1}{\Gamma(1-\beta)} \frac{d}{d t} \int_{0}^{t} \frac{f_{1}(x, \tau)}{(t-\tau)^{\beta}} d \tau\right|_{x=\gamma(t)} .
$$

We first calculate the derivative:

$$
\begin{aligned}
& J(t, x, \beta)=\frac{d}{d t} \int_{0}^{t} \frac{1}{(t-\tau)^{\beta}}\left(\int_{0}^{\tau} \operatorname{erf}\left(\frac{x}{2 \sqrt{\tau-\theta}}\right) \mu(\theta) d \theta\right) d \tau=\left\|\begin{array}{c}
0 \leq \theta \leq \tau \\
0 \leq \tau \leq t
\end{array}\right\|= \\
& =\frac{d}{d t} \int_{0}^{t} \mu(\theta)\left(\int_{0}^{t} \frac{1}{(t-\tau)^{\beta}} \operatorname{erf}\left(\frac{x}{2 \sqrt{\tau-\theta}}\right) d \tau\right) d \theta=\frac{d}{d t} \int_{0}^{t} \mu(\theta) I(x, t, \theta, \beta) d \theta
\end{aligned}
$$

where

$$
I(x, t, \theta, \beta)=\int_{0}^{t} \frac{1}{(t-\tau)^{\beta}} \operatorname{erf}\left(\frac{x}{2 \sqrt{\tau-\theta}}\right) d \tau .
$$

We calculate $I(x, t, \theta, \beta)$.

$$
\begin{gathered}
I(x, t, \theta, \beta)=\int_{\theta}^{t} \frac{1}{(t-\tau)^{\beta}} \frac{2}{\sqrt{\pi}} \int_{0}^{\frac{x}{2 \sqrt{t-\theta}}} e^{-z^{2}} d z d \tau= \\
=\frac{2}{\sqrt{\pi}} \int_{0}^{\frac{x}{2 \sqrt{t-\theta}}} \int_{0}^{t} \frac{1}{(t-\tau)^{\beta}} e^{-z^{2}} d \tau d z+\frac{2}{\sqrt{\pi}} \int_{\frac{x}{2 \sqrt{t-\theta}}}^{+\infty} \int_{\theta}^{\theta+\frac{x^{2}}{4 z^{2}}} \frac{1}{(t-\tau)^{\beta}} e^{-z^{2}} d \tau d z= \\
=\frac{2}{\sqrt{\pi}(\beta-1)} \int_{0}^{\frac{x}{2 \sqrt{t-\theta}}} e^{-z^{2}}\left(0-(t-\theta)^{1-\beta}\right) d z+\frac{2}{\sqrt{\pi}(\beta-1)} \int_{\frac{x}{2 \sqrt{t-\theta}}}^{+\infty} e^{-z^{2} \times} \\
\times\left(\left(t-\theta-\frac{x^{2}}{4 z^{2}}\right)-(t-\theta)^{1-\beta}\right) d z=\frac{2(t-\theta)^{1-\beta}}{\sqrt{\pi}(1-\beta)} \int_{0}^{\frac{x}{2 \sqrt{t-\theta}}} e^{-z^{2}} d z+
\end{gathered}
$$




$$
\begin{gathered}
+\frac{2(t-\theta)^{1-\beta}}{\sqrt{\pi}(1-\beta)} \int_{\frac{x}{2 \sqrt{t-\theta}}}^{+\infty} e^{-z^{2}} d z+\frac{2}{\sqrt{\pi}(\beta-1)} \int_{\frac{x}{2 \sqrt{t-\theta}}}^{+\infty} e^{-z^{2}}\left(t-\theta-\frac{x^{2}}{4 z^{2}}\right)^{1-\beta} d z= \\
=\frac{(t-\theta)^{1-\beta}}{1-\beta}+\frac{2}{\sqrt{\pi}(\beta-1)} I_{1}(x, t, \theta, \beta),
\end{gathered}
$$

where

$$
\begin{gathered}
I_{1}(x, t, \theta, \beta)=\int_{\frac{x}{2 \sqrt{t-\theta}}}^{+\infty} e^{-z^{2}}\left(t-\theta-\frac{x^{2}}{4 z^{2}}\right)^{1-\beta} d z= \\
=(t-\theta)^{1-\beta} \int_{\frac{x}{2 \sqrt{t-\theta}}}^{+\infty} e^{-z^{2}} z^{2 \beta-2}\left(z^{2}-\left(\frac{x}{2 \sqrt{t-\theta}}\right)^{2}\right)^{1-\beta} d z=\left\|\xi=z^{2}\right\|= \\
=\frac{(t-\theta)^{1-\beta}}{2} \int_{\frac{x^{2}}{4(t-\theta)}}^{+\infty} e^{-\xi} \xi^{\beta-\frac{3}{2}}\left(\xi-\frac{x^{2}}{4(t-\theta)}\right)^{1-\beta} d \xi .
\end{gathered}
$$

To calculate the last integral, we use the formula 2.3 .4 (6) from [21; 261]. Then we obtain

$$
I_{1}(x, t, \theta, \beta)=\frac{(t-\theta)^{1-\beta}}{2} \Gamma(2-\beta) \frac{x}{2 \sqrt{t-\theta}} \exp \left(-\frac{x^{2}}{4(t-\theta)}\right) \Psi\left(2-\beta ; \frac{3}{2} ; \frac{x^{2}}{4(t-\theta)}\right),
$$

where $\Psi(a, c ; z)$ is Tricomi degenerate hypergeometric function [18; 1072].

Substituting (19) into (18), we get an expression for (17)

$$
I(x, t, \theta, \beta)=\frac{(t-\theta)^{1-\beta}}{1-\beta}-\frac{x \Gamma(1-\beta)(t-\theta)^{\frac{1}{2}-\beta}}{2 \sqrt{\pi}} \exp \left(-\frac{x^{2}}{4(t-\theta)}\right) \Psi\left(2-\beta ; \frac{3}{2} ; \frac{x^{2}}{4(t-\theta)}\right) .
$$

By virtue of the asymptotic formula (5) (as $t \rightarrow \tau$ ) and

$$
\lim _{t \rightarrow \tau}(t-\tau)^{\frac{1}{2}-\beta} \exp \left(-\frac{x^{2}}{4(t-\tau)}\right)=0
$$

after differentiation operation equality (16) can be rewritten as:

$$
\begin{aligned}
& J(t ; x ; \beta)=\int_{0}^{t} \mu(t) \frac{d}{d t}\left[\frac{(t-\tau)^{1-\beta}}{1-\beta}-\frac{x \Gamma(1-\beta)(t-\tau)^{\frac{1}{2}-\beta}}{2 \sqrt{\pi}} \times\right. \\
& \left.\quad \times \exp \left(-\frac{x^{2}}{4(t-\tau)}\right) \Psi\left(2-\beta ; \frac{3}{2} ; \frac{x^{2}}{4(t-\tau)}\right)\right] d \tau .
\end{aligned}
$$

Let us introduce the notation $z=\frac{x^{2}}{4(t-\tau)} \Rightarrow t-\tau=\frac{x^{2}}{4 z}$.

Then

$$
(t-\tau)^{\frac{1}{2}-\beta} \exp \left(-\frac{x^{2}}{4(t-\tau)}\right) \Psi\left(2-\beta ; \frac{3}{2} ; \frac{x^{2}}{4(t-\tau)}\right)=\frac{x^{1-2 \beta}}{2^{1-2 \beta}} z^{\beta-\frac{1}{2}} \exp (-z) \Psi\left(2-\beta ; \frac{3}{2} ; z\right) .
$$

To calculate the derivative in the second term of equality (20), we use formula (41) from [19; 368] when $n=1$ :

$$
\begin{gathered}
\frac{d}{d t}\left((t-\tau)^{\frac{1}{2}-\beta} \exp \left(-\frac{x^{2}}{4(t-\tau)}\right) \Psi\left(2-\beta ; \frac{3}{2} ; \frac{x^{2}}{4(t-\tau)}\right)\right)= \\
=\frac{x^{1-2 \beta}}{2^{1-2 \beta}} \frac{d}{d z}\left(z^{\beta-\frac{1}{2}} \exp (-z) \Psi\left(2-\beta ; \frac{3}{2} ; z\right)\right) \frac{d z}{d t}= \\
=-\frac{x^{1-2 \beta}}{2^{1-2 \beta}} z^{\beta-\frac{3}{2}} \exp (-z) \Psi\left(1-\beta ; \frac{3}{2} ; z\right)\left(-\frac{x}{4(t-\tau)^{2}}\right)= \\
=\frac{1}{(t-\tau)^{\beta+\frac{1}{2}}} \exp \left(-\frac{x^{2}}{4(t-\tau)}\right) \Psi\left(1-\beta ; \frac{3}{2} ; \frac{x^{2}}{4(t-\tau)}\right) .
\end{gathered}
$$

Mathematics series. № 1(101)/2021 
Then expression (20) can be rewritten as:

$$
J(t ; x ; \beta)=\int_{0}^{t} \mu(\tau)\left[\frac{1}{(t-\tau)^{\beta}}-\frac{x \Gamma(1-\beta)}{2 \sqrt{\pi}(t-\tau)^{\beta+\frac{1}{2}}} \exp \left(-\frac{x^{2}}{4(t-\tau)}\right) \Psi\left(1-\beta ; \frac{3}{2} ; \frac{x^{2}}{4(t-\tau)}\right)\right] d \tau
$$

Now from (13) after taking the fractional derivative of order $\beta$ and substituting $x=\gamma(t)$ taking into account the notation (14) and (21) we obtain the integral equation:

$$
\begin{gathered}
\mu(t)+\frac{\lambda}{\Gamma(1-\beta)} \int_{0}^{t} \frac{\mu(\tau)}{(t-\tau)^{\beta}} d \tau-\frac{\lambda}{2 \sqrt{\pi}} \int_{0}^{t} \frac{\gamma(t)}{(t-\tau)^{\beta+\frac{1}{2}}} \exp \left(-\frac{\gamma^{2}(t)}{4(t-\tau)}\right) \times \\
\times \Psi\left(1-\beta, \frac{3}{2} ; \frac{\gamma^{2}(t)}{4(t-\tau)}\right) \mu(\tau) d \tau=f_{2}(t) .
\end{gathered}
$$
kind:

So, the boundary value problem (10)-(11) has been reduced to the Volterra integral equation of the second

$$
\mu(t)+\lambda \int_{0}^{t} K_{\beta}(t, \tau) \mu(\tau) d \tau=f_{2}(t)
$$

with the right-hand side $f_{2}(t)$, defined by formula (15), and the kernel

$$
K_{\beta}(t, \tau)=\frac{1}{\Gamma(1-\beta)(t-\tau)^{\beta}}-\frac{\gamma(t)}{2 \sqrt{\pi}(t-\tau)^{\beta+\frac{1}{2}}} \exp \left(-\frac{\gamma^{2}(t)}{4(t-\tau)}\right) \Psi\left(1-\beta, \frac{3}{2} ; \frac{\gamma^{2}(t)}{4(t-\tau)}\right)
$$

where $\Psi(a, b, y)$ is the Tricomi degenerate hypergeometric function that can be represented as an integral (4).

\section{Continuity in the order of the derivative in the loaded term of the problem}

Lemma 2. For boundary value problem (10)-(11) there is continuity in the order $\beta$ of the derivative in the loaded term of equation (10).

Proof. We consider the limiting cases for the fractional derivative order of the term with the load in the equation (10).

I. $\quad \beta=0$. Then from (2) and (3) we have

$$
\left.D_{0, t}^{0} u(x, t)\right|_{x=\gamma(t)}=\left.u(x, t)\right|_{x=\gamma(t)}=u(\gamma(t), t) .
$$

From (10)-(11) we get a boundary value problem when $\beta=0$ :

$$
\left\{\begin{array}{c}
u_{t}-u_{x x}+\lambda \mu(t)=f(x, t) \\
u(x, 0)=0 ; \quad u(0, t)=0
\end{array},\right.
$$

where $\mu(t)=u(\gamma(t), t)$.

We write down its solution inverting the differential part by formula (8):

$$
u(x, t)=-\lambda \int_{0}^{t} \operatorname{erf}\left(\frac{x}{2 \sqrt{t-\tau}}\right) \mu(\tau) d \tau+f_{1}(x, t),
$$

where

$$
f_{1}(x, t)=\int_{0}^{t} \int_{0}^{\infty} G(x, \xi, t-\tau) f(\xi, \tau) d \xi d \tau .
$$

When $x=\gamma(t)$ taking into account the notation $u(\gamma(t), t)=\mu(t)$ from (24) we obtain the Volterra integral equation of the second kind:

$$
\mu(t)+\lambda \int_{0}^{t} \operatorname{erf}\left(\frac{\gamma(t)}{2 \sqrt{t-\tau}}\right) \mu(\tau) d \tau=f_{1}(t)
$$

where $f_{2}(t)=f_{1}(\gamma(t), t)$.

Now we find $\lim _{\beta \rightarrow 0+0}$ from (23). 
The function under the limit sign is definite and continuous for $\beta=0$, therefore, we can make the passage to the limit taking into account formula (4) and formula 2.3.4 (5) [21; 260]:

$$
\begin{aligned}
\lim _{\beta \rightarrow 0+0} K_{\beta}(t, \tau) & =1-\frac{\gamma(t)}{2 \sqrt{\pi} \sqrt{t-\tau}} \exp \left(-\frac{\gamma^{2}(t)}{4(t-\tau)}\right) \Psi\left(1 ; \frac{3}{2} ; \frac{\gamma^{2}(t)}{4(t-\tau)}\right)= \\
=1-\frac{\gamma(t)}{2 \sqrt{\pi} \sqrt{t-\tau}} & \exp \left(-\frac{\gamma^{2}(t)}{4(t-\tau)}\right) \int_{0}^{\infty}(1+\xi)^{-\frac{1}{2}} \exp \left(-\frac{\gamma^{2}(t)}{4(t-\tau)} \xi\right) d \xi= \\
= & 1-\operatorname{erfc}\left(\frac{\gamma(t)}{2 \sqrt{t-\tau}}\right)=\operatorname{erf}\left(\frac{\gamma(t)}{2 \sqrt{t-\tau}}\right) .
\end{aligned}
$$

So, $\lim _{\beta \rightarrow 0+0} K_{\beta}(t, \tau)=\operatorname{erf}\left(\frac{\gamma(t)}{2 \sqrt{t-\tau}}\right)$. Then equation (22) coincides with equation (25) for $\beta=0$.

II. $\beta=1$. Then from (2) and (3) we have

$$
\left.D_{0, t}^{1} u(x, t)\right|_{x=\gamma(t)}=\left.\frac{d u(x, t)}{d t}\right|_{x=\gamma(\tau)}=\left.u_{t}(x, t)\right|_{x=\gamma(t)} .
$$

From (10)-(11) we get a boundary value problem when $\beta=1$ :

$$
\left\{\begin{array}{c}
u_{t}-u_{x x}+\lambda \mu(t)=f(x, t) \\
u(x, 0)=0 ; \quad u(0, t)=0
\end{array}\right.
$$

where $\mu(t)=\left.u_{t}(x, t)\right|_{x=\gamma(t)}$.

We write down its solution inverting the differential part by formula (8):

$$
u(x, t)=-\lambda \int_{0}^{t} \operatorname{erf}\left(\frac{x}{2 \sqrt{t-\tau}}\right) \mu(\tau) d \tau+f_{1}(x, t),
$$

where the function $f_{1}(x, t)$ is defined by formula (12).

We calculate the derivative with respect to $t$ of $(26)$ :

$$
u_{t}(x, t)=-\lambda\left\{\mu(t)+\int_{0}^{t} \frac{2}{\sqrt{\pi}} \exp \left(-\frac{x^{2}}{4(t-\tau)}\right)\left(-\frac{x}{4(t-\tau)^{3 / 2}}\right) \mu(\tau) d \tau\right\}+f_{1 t}(x, t) .
$$

Substituting $x=\gamma(t)$ and taking into account the notation $\left.u_{t}(x, t)\right|_{x=\gamma(t)}=\mu(t)$ we obtain the Volterra integral equation of the second kind:

$$
\mu(t)-\frac{\lambda}{1+\lambda} \int_{0}^{t} \frac{\gamma(t)}{2 \sqrt{\pi}(t-\tau)^{3 / 2}} \exp \left(-\frac{\gamma^{2}(t)}{4(t-\tau)}\right) \mu(\tau) d \tau=f_{2}(t)
$$

where $f_{2}(t)=\frac{1}{1+\lambda} f_{1 t}(\gamma(t), t)$.

Taking into account formula (4) and the well-known relation $\lim _{\alpha \rightarrow 0} \Gamma(\alpha)=\infty$ when taking the limit at $\beta \rightarrow 1-0$ from (23) we get:

$$
\begin{gathered}
\lim _{\beta \rightarrow 1-0} K_{\beta}(t, \tau)=-\frac{\gamma(t)}{2 \sqrt{\pi(t-\tau)^{3}}} \exp \left(-\frac{\gamma^{2}(t)}{4(t-\tau)}\right) \times \\
\times \lim _{\beta \rightarrow 1-0} \frac{1}{\Gamma(1-\beta)} \int_{0}^{\infty} \xi^{-\beta}(1+\xi)^{\beta-\frac{1}{2}} \exp \left(-\frac{\gamma^{2}(t)}{4(t-\tau)} \xi\right) d \xi .
\end{gathered}
$$

To calculate the last integral, we use the formula (12) from [21; 262].

Then the limit relation (28) can be rewritten as:

$$
\begin{gathered}
\lim _{\beta \rightarrow 1-0} K_{\beta}(t, \tau)=-\frac{1}{\sqrt{\pi}(t-\tau)} \exp \left(-\frac{\gamma^{2}(t)}{8(t-\tau)}\right) \lim _{\beta \rightarrow 1-0} \frac{2^{1 / 2-\beta} \Gamma(1-\beta)}{\Gamma(1-\beta)} D_{2 \beta-1}\left(\frac{\gamma(t)}{\sqrt{2(t-\tau)}}\right)= \\
=-\frac{2^{-\frac{1}{2}}}{\sqrt{\pi}(t-\tau)} \exp \left(-\frac{\gamma^{2}(t)}{8(t-\tau)}\right) D_{1}\left(\frac{\gamma(t)}{\sqrt{2(t-\tau)}}\right)=-\frac{\gamma(t)}{2 \sqrt{\pi}(t-\tau)^{3 / 2}} \exp \left(-\frac{\gamma^{2}(t)}{4(t-\tau)}\right) .
\end{gathered}
$$


Here, for the parabolic cylinder function $D_{2 \beta-1}(z)$, we used formula 9.251 from $[18 ; 1080]$ when $\beta=1$ :

$$
D_{1}(z)=-e^{\frac{z^{2}}{4}} \frac{d}{d z}\left(e^{-\frac{z^{2}}{2}}\right)=z e^{-\frac{z^{2}}{4}} .
$$

So for equation $(22)$

$$
\lim _{\beta \rightarrow 1} K_{\beta}(t, \tau)=-\frac{\gamma(t)}{2 \sqrt{\pi}(t-\tau)^{3 / 2}} \exp \left(-\frac{\gamma^{2}(t)}{4(t-\tau)}\right) .
$$

The obtained result coincides with the kernel of integral equation (27).

Lemma 2 is completely proved.

5 Connection of the singularities of the integral equation kernel with the fractional derivative order in the problem loaded term and with the load behavior. Main result

To establish the main result of the paper we investigate kernel (23) of integral equation (22), which has singularities for $\tau=t$ and $t=0$.

Direct investigation of kernel (23) is difficult, since the kernel contains the degenerate hypergeometric Tricomi function. Therefore we find

$$
\lim _{t \rightarrow 0+0} \int_{0}^{t} K_{\beta}(t, \tau) d \tau \text {. }
$$

Theorem. Integral equation (22) with kernel (23) for $0 \leq \beta<1$ and with $\gamma(t) \sim t^{\omega}$ in the neighborhood of $t=0$ is uniquely solvable in the class of continuous functions for any continuous right-hand side $f_{2}(t)$ defined by formula (15), if $\frac{1}{2} \leq \omega<1-2 \beta$ or $0 \leq \omega<\frac{1}{2}, \quad 0 \leq \beta \leq 1$.

Proof. We have

$$
\begin{gathered}
\int_{0}^{t} K_{\beta}(t, \tau) d \tau=\frac{1}{\Gamma(1-\beta)} \int_{0}^{t} \frac{d \tau}{(t-\tau)^{\beta}}- \\
-\frac{\gamma(t)}{2 \sqrt{\pi}} \int_{0}^{t} \frac{1}{(t-\tau)^{\beta+\frac{1}{2}}} \exp \left(-\frac{\gamma^{2}(t)}{4(t-\tau)}\right) \Psi\left(1-\beta ; \frac{3}{2} ; \frac{\gamma^{2}(t)}{4(t-\tau)}\right) d \tau
\end{gathered}
$$

and when $0 \leq \beta<1$ :

$$
\int_{0}^{t} \frac{d \tau}{(t-\tau)^{\beta}}=\frac{1}{1-\beta} t^{1-\beta}
$$

To calculate the integral in the 2 nd term of expression (29), we use the representation of the Tricomi function in terms of the Whittaker function by formula (7):

$$
\Psi\left(1-\beta ; \frac{3}{2} ; \frac{\gamma^{2}(t)}{4(t-\tau)}\right)=\left(\frac{\gamma^{2}(t)}{4(t-\tau)}\right)^{-\frac{3}{4}} \exp \left(\frac{\gamma^{2}(t)}{8(t-\tau)}\right) W_{\beta-\frac{1}{4} ; \frac{1}{4}}\left(\frac{\gamma^{2}(t)}{4(t-\tau)}\right)
$$

Then the integral in the 2nd term of the expression (29) takes the form

$$
\begin{gathered}
I(t, \beta)=\int_{0}^{t} \frac{1}{(t-\tau)^{\beta+\frac{1}{2}}} \exp \left(-\frac{\gamma^{2}(t)}{4(t-\tau)}\right) \Psi\left(1-\beta ; \frac{3}{2} ; \frac{\gamma^{2}(t)}{4(t-\tau)}\right) d \tau= \\
=\frac{2^{3 / 2}}{(\gamma(t))^{3 / 2}} \int_{0}^{t}(t-\tau)^{\frac{1}{4}-\beta} \exp \left(-\frac{\gamma^{2}(t)}{8(t-\tau)}\right) W_{\beta-\frac{1}{4} ; \frac{1}{4}}\left(\frac{\gamma^{2}(t)}{4(t-\tau)}\right) d \tau= \\
=\frac{2^{2 \beta-1}}{(\gamma(t))^{2 \beta-1}} \int_{\frac{\gamma^{2}(t)}{4 t}}^{+\infty} z^{\beta-\frac{9}{4}} e^{-\frac{z}{2}} W_{\beta-\frac{1}{4} ; \frac{1}{4}}(z) d z=\frac{2^{3 / 2} t^{\frac{5}{4}-\beta}}{(\gamma(t))^{3 / 2}} \exp \left(-\frac{\gamma^{2}(t)}{8 t}\right) W_{\beta-\frac{5}{4} ; \frac{1}{4}}\left(\frac{\gamma^{2}(t)}{4 t}\right) .
\end{gathered}
$$

In the calculation, we have introduced the replacement $z=\frac{\gamma^{2}(t)}{4(t-\tau)}$ and used formula 2.19.5 (13) from [19; $217]$.

Let $\gamma(t) \sim t^{\omega}$ when $t \rightarrow 0+0$. Then (31) can be rewritten as (in the neighborhood of the point $t=0$ ):

$$
I(t ; \beta)=2^{\frac{3}{2}} t^{\frac{5}{4}-\beta-\frac{3}{2} \omega} \exp \left(-\frac{1}{8} t^{2 \omega-1}\right) W_{\beta-\frac{5}{4} ; \frac{1}{4}}\left(\frac{1}{4} t^{2 \omega-1}\right) .
$$


For Whittaker function, we use formula 7.2.2 (5) from [19; 366]:

$$
W_{\beta-\frac{5}{4} ; \frac{1}{4}}\left(\frac{1}{4} t^{2 \omega-1}\right)=2^{-\frac{3}{2}} t^{\frac{1}{2} \omega-\frac{3}{4}} \exp \left(-\frac{1}{8} t^{2 \omega-1}\right) \Psi\left(2-\beta ; \frac{3}{2} ; \frac{1}{4} t^{2 \omega-1}\right) .
$$

Then

$$
I(t ; \beta)=t^{\frac{1}{2}-\beta-\omega} \exp \left(-\frac{1}{4} t^{2 \omega-1}\right) \Psi\left(2-\beta ; \frac{3}{2} ; \frac{1}{4} t^{2 \omega-1}\right) .
$$

For Tricomi function $\Psi(a, b, z)$, we use representation (4) and then we apply formula (12) from [21; 262]:

$$
\begin{gathered}
I(t ; \beta)=\frac{t^{\frac{1}{2}-\beta-\omega}}{\Gamma(2-\beta)} \exp \left(-\frac{1}{4} t^{2 \omega-1}\right) \int_{0}^{\infty} \xi^{1-\beta}(1+\xi)^{\beta-\frac{3}{2}} \exp \left(-\frac{1}{4} t^{2 \omega-1} \xi\right) d \xi= \\
=2^{\frac{5}{2}-\beta} t^{1-2 \beta-2 \omega} \exp \left(-\frac{1}{8} t^{2 \omega-1}\right) D_{2 \beta-3}\left(\frac{1}{\sqrt{2}} t^{\omega-\frac{1}{2}}\right) .
\end{gathered}
$$

So, substituting (30) and (32) into (29), we get $0 \leq \beta<1$ when $\quad t \rightarrow 0$ :

$$
\int_{0}^{t} K_{\beta}(t, \tau) d \tau=\frac{t^{1-\beta}}{\Gamma(2-\beta)}-\frac{2^{\frac{3}{2}-\beta}}{\sqrt{\pi}} t^{1-2 \beta-\omega} \exp \left(-\frac{1}{8} t^{2 \omega-1}\right) D_{2 \beta-3}\left(\frac{1}{\sqrt{2}} t^{\omega-\frac{1}{2}}\right) .
$$

Cases are possible (when $0 \leq \beta<1$ ):

1) $2 \omega-1>0$. If $0 \leq \beta<1$, then $-1-2 \beta-\omega<\frac{1}{2}$.

We first calculate $D_{2 \beta-3}(0)$ using formula 8.3 (1) from [22; 125] and formula 194 (3) from [18; 299].

$$
D_{2 \beta-3}(0)=\frac{2^{\beta-\frac{3}{2}}}{\Gamma\left(\frac{3}{2}-\beta\right)} \int_{0}^{\infty} t^{\frac{1}{2}-\beta}(1+t)^{\beta-2} d t=\frac{2^{\beta-\frac{3}{2}}}{\Gamma\left(\frac{3}{2}-\beta\right)} B\left(\frac{3}{2}-\beta ; \frac{1}{2}\right)=\frac{2^{\beta-\frac{3}{2}} \sqrt{\pi}}{\Gamma(2-\beta)} .
$$

Then from (33) when $\omega>\frac{1}{2}$ and $1-2 \beta-\omega \geq 0 \quad(0 \leq \beta<1)$ we have:

$$
\lim _{t \rightarrow 0} \int_{0}^{t} K_{\beta}(t, \tau) d \tau= \begin{cases}0 ; & \text { if } 1-2 \beta-\omega>0, \quad \omega>\frac{1}{2}, \quad 0 \leq \beta<1 ; \\ -\frac{1}{\Gamma(2-\beta)} ; & \text { if } 1-2 \beta-\omega=0, \quad \omega>\frac{1}{2}, \quad 0 \leq \beta<1 \\ \infty ; & \text { if } 1-2 \beta-\omega<0, \quad \omega>\frac{1}{2}, \quad 0 \leq \beta<1 .\end{cases}
$$

We consider the case $\beta=1$. It was shown above that

$$
K_{1}(t, \tau)=-\frac{\gamma(t)}{2 \sqrt{\pi}(t-\tau)^{3 / 2}} \exp \left(-\frac{\gamma^{2}(t)}{4(t-\tau)}\right) .
$$

If $\gamma(t) \sim t^{\omega}$ at $t \rightarrow 0+0$ then

$$
\int_{0}^{t} K_{1}(t, \tau) d \tau=-\int_{0}^{t} \frac{t^{\omega}}{2 \sqrt{\pi}(t-\tau)^{3 / 2}} \exp \left(-\frac{t^{2 \omega}}{4(t-\tau)}\right) d \tau .
$$

After introducing the replacement

$$
\begin{aligned}
& z=\sqrt{\frac{t^{2 \omega}}{4(t-\tau)}} ; \quad t-\tau=\frac{t^{2 \omega}}{4 z^{2}} ; \quad d \tau=\frac{t^{2 \omega}}{2 z^{3}} d z \\
& \tau=0 \Rightarrow z=\frac{1}{2} t^{\frac{2 \omega-1}{2}}, \quad \tau=t \Rightarrow z \rightarrow+\infty
\end{aligned}
$$

integral (35) takes the form:

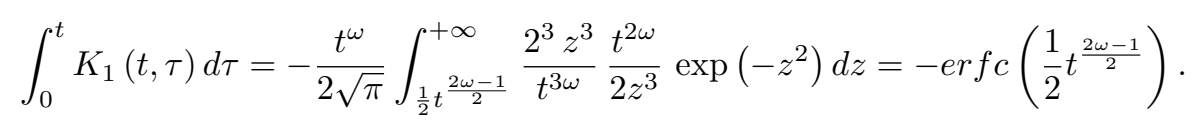

Then

$$
\lim _{t \rightarrow 0+0} \int_{0}^{t} K_{1}(t, \tau) d \tau= \begin{cases}-1 ; & \text { if } \quad \omega>\frac{1}{2} \\ 0 ; & \text { if } \quad 0 \leq \omega<\frac{1}{2} .\end{cases}
$$


By the condition stated, the function $\gamma(t)$ increases in the domain $\bar{Q}$ and $\gamma(0)=0$. Therefore, the case $\omega<0$ is not considered.

Consider the case $0<\omega<\frac{1}{2}$ and $0 \leq \beta<1$. Then in (33) argument of the parabolic cylinder function $\rightarrow+\infty$ if $t \rightarrow 0+0$. Since there is an asymptotic expansion of the function (see formula 9.246 (1) [18; 1079]) we obtain

$$
\lim _{t \rightarrow 0+0} \int_{0}^{t} K_{\beta}(t, \tau) d \tau=\lim _{t \rightarrow 0+0} \frac{t^{1-\beta}}{\Gamma(2-\beta)}-\frac{1}{\sqrt{\pi}} \lim _{t \rightarrow 0+0} t^{\frac{3}{2}-2 \omega(1-\beta)-3 \beta} \exp \left(-\frac{1}{4 t^{\frac{1}{2}-\omega}}\right)=0 .
$$

It remains to investigate the case $\omega=\frac{1}{2}$ for different values $\beta$.

Let be $0 \leq \beta<1$. For $x=\gamma(t)=\sqrt{t}$ equation (22) has the kernel:

$$
K_{\beta}(t, \tau)=\frac{1}{\Gamma(1-\beta)(t-\tau)^{\beta}}-\frac{\sqrt{t}}{2 \sqrt{\pi}(t-\tau)^{\beta+\frac{1}{2}}} \exp \left(-\frac{t}{4(t-\tau)}\right) \Psi\left(1-\beta ; \frac{3}{2} ; \frac{t}{4(t-\tau)}\right)
$$

and the right side according to formula (15) when $x=\sqrt{t}$. Kernel (38) has singularities at $\tau=t$ and $t=0$. We find

$$
\int_{0}^{t} K_{\beta}(t, \tau) d \tau=\frac{t^{1-\beta}}{\Gamma(2-\beta)}-\frac{\sqrt{t}}{2 \sqrt{\pi}} \int_{0}^{t} \frac{1}{(t-\tau)^{\beta+\frac{1}{2}}} \exp \left(-\frac{t}{4(t-\tau)}\right) \Psi\left(1-\beta ; \frac{3}{2} ; \frac{t}{4(t-\tau)}\right) d \tau .
$$

Repeating the above calculations, we get a formula similar to (33), when $0 \leq \beta<1$

$$
\int_{0}^{t} K_{\beta}(t, \tau) d \tau=\frac{t^{1-\beta}}{\Gamma(2-\beta)}-\frac{2^{\frac{1}{2}-\beta}}{\sqrt{\pi}} t^{\frac{1}{2}-2 \beta} \exp \left(-\frac{1}{8}\right) D_{2 \beta-3}\left(\frac{1}{\sqrt{2}}\right) .
$$

We calculate $D_{2 \beta-3}\left(\frac{1}{\sqrt{2}}\right)$ using formula 9.241 (1) from [18; 1078].

$$
D_{2 \beta-3}\left(\frac{1}{\sqrt{2}}\right)=\frac{1}{\sqrt{\pi}} 2^{2 \beta-\frac{1}{2}} e^{-\frac{\pi}{2}(2 \beta-1) i} e^{\frac{1}{8}} \int_{-\infty}^{+\infty} x^{2 \beta-1} e^{-2 x^{2}+\frac{x}{\sqrt{2}} i} d x .
$$

To calculate the integral

$$
J(\beta)=\int_{-\infty}^{+\infty} x^{2 \beta-1} e^{-2 x^{2}+\frac{x}{\sqrt{2}} i} d x
$$

we use the formula $3.462(3)$ from $[18 ; 352]$

$$
J(\beta)=\frac{1}{i^{2 \beta-1}} \int_{-\infty}^{+\infty}(i x)^{2 \beta-1} e^{-2 x^{2}-i \frac{x}{\sqrt{2}}} d x=\frac{2^{\frac{1}{2}-2 \beta}}{i^{2 \beta-1}} \sqrt{\pi} e^{-\frac{1}{32}} D_{2 \beta-3}\left(\frac{1}{2 \sqrt{2}}\right) .
$$

Then

$$
D_{2 \beta-3}\left(\frac{1}{\sqrt{2}}\right)=\frac{1}{i^{2 \beta-2}} \sin \left(\frac{\pi}{2}-\pi \beta\right) D_{2 \beta-3}\left(\frac{1}{2 \sqrt{2}}\right) .
$$

Note that the argument of the parabolic cylinder function decreases exponentially with the denominator $\frac{1}{2}$, remaining positive. It was previously calculated that

$$
D_{2 \beta-3}(0)=\frac{2^{\beta-\frac{3}{2}} \sqrt{\pi}}{\Gamma(2-\beta)}
$$

So we obtain that $D_{2 \beta-3}\left(\frac{1}{\sqrt{2}}\right)$ is a finite constant depending on $\beta$.

Then from (39) we have (when $0 \leq \beta<1$ and $\omega=\frac{1}{2}$ ):

$$
\lim _{t \rightarrow 0+0} \int_{0}^{t} K_{1}(t, \tau) d \tau= \begin{cases}0 ; & \text { if } \quad 0 \leq \beta<\frac{1}{4} \\ \text { const } \neq 0 ; & \text { if } \beta=\frac{1}{4} \\ \infty ; & \text { if } \frac{1}{4}<\beta<1\end{cases}
$$


Now consider the case for $\gamma(t)=\sqrt{t}$ and $\beta=1$ :

$$
\begin{aligned}
\int_{0}^{t} K_{1}(t, \tau) d \tau & =-\frac{\sqrt{t}}{2 \sqrt{\pi}} \int_{0}^{t} \frac{d \tau}{(t-\tau)^{\frac{3}{2}}} \exp \left(-\frac{t}{4(t-\tau)}\right) d \tau=\left\|\begin{array}{c}
z=\sqrt{t-\tau}, \tau=t-z^{2}, \quad \|= \\
d \tau=-2 z d z
\end{array}\right\| \\
& =-\frac{\sqrt{t}}{\sqrt{\pi}} \int_{0}^{\sqrt{t}} \frac{1}{z^{2}} e^{-\frac{t}{4 z^{2}}} d z=-\frac{2}{\sqrt{\pi}} \int_{\frac{1}{2}}^{+\infty} e^{-\xi^{2}} d \xi=-\operatorname{erfc}\left(\frac{1}{2}\right) .
\end{aligned}
$$

Then

$$
\lim _{t \rightarrow 0} \int_{0}^{t} K_{1}(t, \tau) d \tau=-\operatorname{erfc}\left(\frac{1}{2}\right) \neq 0
$$

if $\gamma(t)=\sqrt{t}$ and $\beta=1$.

Summarizing results (34)-(41), we get the main result. The theorem is completely proved.

\section{Conclusions}

Under the conditions of the theorem, kernel (23) of the integral equation has a weak singularity. Therefore, the method of successive approximations can be used to find a unique solution to the equation (22) in the class of continuous functions. And the corresponding boundary value problems are well-posed in natural classes of functions, i.e. loaded term is a weak perturbation.

If $\omega \geq \frac{1}{2}$ and $\omega \geq 1-2 \beta$ when $0 \leq \beta \leq 1$ for $\gamma(t) \sim t^{\omega}$ at $t \rightarrow 0+0$ integral equation (22) is not solvable by the method of successive approximations. It can be shown that the corresponding homogeneous equation for some values of the parameter $\lambda$ will have nonzero solutions. If the uniqueness of the solution to the first boundary value problem is violated, then in this case the load can be interpreted as a strong perturbation. So, the existence and uniqueness of solutions to the integral equation depends on the order of the fractional derivative in the loaded term.

\section{Acknowledgments}

This research was funded by the Science Committee of the Ministry of Education and Science of the Republic of Kazakhstan (Grant No. AP08955795, 2020-2022.)

\section{References}

1 Samko, S. G., Kilbas, A. A., \& Marichev, O. I. (1993). Fractional Integrals and Derivatives. Theory and Application. Gordon and Breach: New York, 1006 p.

2 Le Mehaute, A., Tenreiro Machado, J. A., Trigeassou, J. C., \& Sabatier, J. (2005). Fractional Differentiation and its Applications. Bordeaux Univ: Bordeaux.

3 Podlubny, I. (2002). Geometric and physical interpretation of fractional integration and fractional differentiation. Fract. Calculus Appl. Anal., 5, 367-386. an: 1042.26003.

4 Heymans, N., \& Podlubny, I. (2006). Physical interpretation of initial conditions for fractional differential equations with Riemann-Liouville fractional derivatives. Rheologica Acta, 45(5), 765-772. DOI: 10.1007 /S00397-005-0043-5.

5 Feng, M., Zhang, X., \& Ge, W. (2011). New existence results for higher-order nonlinear fractional differential equations with integral boundary conditions. Bound. Value Probl. Art., 720702, 20. DOI: $10.1155 / 2011 / 720702$.

6 Cao Labora, Daniel, Rodriguez-Lopez, Rosana, \& Belmekki, Mohammed. (2020). Existence of solutions to nonlocal boundary value problems for fractional differential equations with impulses. Electronic Journal of Differential Equations, 15, 16.

7 Yusuf, A., Qureshi, S., Inc, M., Aliyu, A.I., Baleanu, D., \& Shaikh, A.A. (2018). Two-strain epidemic model involving fractional derivative with Mittag-Leffler kernel. Chaos: An Interdisciplinary Journal of Nonlinear Science, 28(12), 123121. DOI: 10.1063/1.5074084.

8 Nakhushev, A. M. (1983). Loaded equations and their applications. Diff. equations, 19(1), 86-94. 
9 Amangaliyeva, M. M., Akhmanova, D. M., Dzhenaliev, M. T., \& Ramazanov, M. I. (2011). Boundary value problems for a spectrally loaded heat operator with load line approaching the time axis at zero or infinity. Differential Equations, 47(2), 231-243. DOI: 10.1134/S0012266111020091

10 Dzhenaliev, M. T., \& Ramazanov, M. I. (2006). On the boundary value problem for the spectrally loaded heat conduction operator. Siberian Mathematical Journal, 47(3), 433-451. DOI: 10.1007/s11202-0060056-Z

11 Dzhenaliev, M. T., \& Ramazanov, M. I. (2007). On a boundary value problem for a spectrally loaded heat operator: I Differential Equations, 43(4), 513-524. DOI: 10.1134/S0012266107040106

12 Dzhenaliev, M. T., \& Ramazanov, M. I. (2007). On a boundary value problem for a spectrally loaded heat operator: II Differential Equations, 43(6), 806-812. DOI: 10.1134/S0012266107060079

13 Attayev, A. Kh., Iskakov, S. A., Karshigina, G. Zh., \& Ramazanov, M. I. (2014). The first boundary problem for heat conduction equation with a load of fractional order. I. Bulletin of the Karaganda University-mathematics, 4(76), 11-16.

14 Iskakov, S. A., Ramazanov, M. I., \& Ivanov, I. A. (2015). The first boundary problem for heat conduction equation with a load of fractional order. II. Bulletin of the Karaganda University-mathematics, 2(78), 25-30.

15 Kosmakova, M. T., \& Kasymova, L. Zh. (2019). To solving the heat equation with fractional load. Journal of Mathematics, Mechanics and Computer Science, 104(4), 50-62. DOI: 10.26577/JMMCS-2019-4-m6.

Ramazanov, M. I., Kosmakova, M. T., \& Kasymova, L. Zh. (2020). On a Problem of Heat Equation with Fractional Load. Lobachevskii Journal of Mathematics, 41(9), 1873-1885.

DOI: 10.1134/S199508022009022X

16 Luke, Yudell L. (1969). The special functions and their approximations. Academic Press, Inc.: London.

17 Gradshteyn, I.S., \& Ryzhik, I.M. (2007). Table of Integrals, Series, and Products. AP: New York. USA. 7 edition.

18 Prudnikov, A. P., Brychkov, Yu. A., \& Marichev, O. I. (1989). Integrals and Series: More Special Functions. Vol. 3. Gordon and Breach: New York-London.

19 Polyanin, A.D. (2002). Handbook of Linear Partial Differential Equations for Engineers and Scientists. Chapman and Hall/CRC: New York-London.

20 Prudnikov, A. P., Brychkov, Yu. A., \& Marichev, O. I. (1998). Integrals and Series: Elementary Functions. (N.M.Queen, Trans) CRC: New York, USA.

21 Bateman, H., \& Erderlyi, A. (1953). Higher transcendental functions. McGraw-Hill: New York.

\author{
М.Т. Космакова, С.А. Искаков, Л.Ж. Касымова
}

\title{
Жойылатын облыстағы жылуөткізгіштіктің екіөлшемді шекаралық есебінің шешуіне
}

\begin{abstract}
Мақалада үзіліссіз функциялар класындағы жылуөткізгіштіктің бөлшекті-жүктемелі теңдеуі үшін шеттік есеп қарастырылған. Зерттеу әдістері шеттік есептерді интегралдық теңдеулерге келтіруге негізделген зерттеу болып табылады. Қойылған шеттік есеп дифференциалдық бөлікті айналдыру арқылы екінші текті Вольтерра интегралдық теңдеуіне келтірілген. Алынған теңдеудің ядросында арнайы функция бар. Сондай-ақ, жылуөткізгіштік теңдеуінің шеттік есебінің жүктелген қосылғышының бөлшек туындысы ретінің шектік жағдайлары зерттелді. Интегралдық теңдеудің шешуінің бар болуы мен жалғыздығы бастапқы шеттік есептің жүктелген қосылғышындағы бөлшек туындының ретіне байланысты екендігі көрсетілген.
\end{abstract}

Kiлm сөздер: жүктелген теңдеу, бөлшек туынды, жылуөткізгіштік теңдеуі, Вольтерра интегралдық теңдеуі, арнайы функция. 
М.Т. Космакова, С.А. Искаков, Л.Ж. Касымова

\section{К решению двумерной граничной задачи теплопроводности в вырождающейся области}

В статье рассмотрена краевая задача для дробно-нагруженного уравнения теплопроводности в классе непрерывных функций. Методы исследования базируются на подходе к исследованию краевых задач, основанном на их сведении к интегральным уравнениям. Поставленная краевая задача сведена к интегральному уравнению Вольтерра второго рода обращением дифференциальной части. Ядро полученного уравнения содержит специальную функцию. Также проведено исследование предельных случаев порядка дробной производной слагаемого с нагрузкой в уравнении теплопроводности краевой задачи. Показано, что существование и единственность решения интегрального уравнения зависят от порядка дробной производной в нагруженном слагаемом исходной краевой задачи.

Ключевые слова: нагруженное уравнение, дробная производная, уравнение теплопроводности, интегральное уравнение Вольтерра, специальная функция. 